\title{
Improving Directed Diffusion in Sensor Network Using Learning Automata:DDLA New Approach in Directed Diffusion
}

\author{
Ali Sayyad \\ Msc. Information Technology, Network Eng. Computer \\ \& Elec. Department \\ Islamic Azad University of Qazvin \\ Qazvin,Iran \\ later_opt@yahoo.com \\ Mohammad Shojafar \\ Msc. Computer Eng. Computer \& Elec. Department \\ Islamic Azad University of Qazvin \\ Qazvin,Iran \\ Shojafar@qiau.ac.ir
}

\author{
Zia Delkhah \\ Msc. Information Technology, Network Eng. Computer \\ \& Elec. Department \\ Islamic Azad University of Qazvin \\ Qazvin,Iran \\ ziadelkhah@gmail.com \\ Mohammad Reza Meybodi \\ PHD in Computer science. Computer \& IT. Department \\ Amirkabir University,Tehran,Iran \\ mmeybodi@aut.ac.ir
}

\begin{abstract}
One of the important and challenging matters in sensor network is energy of life span of nodes in the network. Node's movement, specifically movement of central node (sink) in these networks cause to increase routing updating overhead and consequently to increase power consumption and to decrease network life span. Directed Diffusion algorithm is one of methods in sensor network which is a data-oriented algorithm. One of the important definitions of basic algorithm is not supporting central node's movement. In case of movement of central node, data packs pass on unreliable rout toward central node. In fact, they pass a rout on which the central node is not present at the time being and it has moved to another place. Therefore, route of data is out of order and there is the need to build new routes. This problem causes to create lots of overhead and waste energy.
\end{abstract}

In this article, it is tried to solve mentioned problem of central node's movement by learning automata. In suggested algorithm by learning automata a route amendment tree is built which prevents from creation of the whole route and its overhead.

Keywords-component; Wireless Sensor Network; Directed Diffusion; Learning Automata; Dynamics

\section{INTRODUCTION}

Current progress in technology of short radio range and micro electro mechanical system leads in production intelligent sensors which are generally calculated by capability of data sense, wireless connection in device is strongly limited with regards to equipped resources. These tiny sensors can be considered for gathering information and they can be distributed to central controlling units named sink in different regions. For transferring sensed data in the network to sink, many wireless steps may be taken through a collection of small and limited sensor nodes with regards to energy. This characteristic is important for diffusing data in wireless sensor network.

Within previous years, different algorithms and protocols were suggested with goal of achieving more efficiency and reliability for diffusing data in wireless sensor networks. For example, flooding diffusion is the most reliable method for sending data from sensor to a sink. This protocol doesn't impose any extra cost for keeping topology and discovery of the route and its implementation is very simple and easy. But, main problem of this protocol is creating too much overhead in the network due to sending repeated messages. This problem specially leads to inefficiency in energy consumption. A new data-oriented method which was different from traditional routing was created for wireless sensor networks in order to solve this problem. The main goal for generating this method is because of concentrating of sensor network on the data generated with sensors and it doesn't focus on sensed nodes. Data-oriented protocols are based on query and are dependent on naming (for instance mentioning data with binary of trait-value) of desirable data. Therefore, too much transfer of many packs is prevented and it causes energy efficiency.

Direct diffusion[1] is an instance of one of data-oriented protocols because of its characteristics for saving energy, such as, data query based on query (named interest) by sinks, collecting and saving data by sensor and mechanism of gradient and route enhancement. Gradient concept usually means direction toward those neighboring by which sink is accessible.

In wireless sensor network, most data packs are sent toward sink from a sensor complex. Therefore, here, each sensor node's important task is to create and keep gradient value in each node. Totally gradient value management is done by primary and frequent primitive flooding diffusion of a series of a controlling pack (like interest packs in direct diffusion [1]) from a sink. Please note that this frequent flooding diffusion all over the network leads in too much overhead (regarding band width and energy consumption) in sensor networks. Moreover, when network topology change because of failure of senor nodes, wireless connections and value of some gradients will become unreliable, thus, there is need to frequent flooding diffusion. This protocol is sensitive to node's movement due to direct diffusion nature. In fact, the best dynamism plan should establish a minimum 
spanning tree which disappears and will appear again in another place for from established routs. The related issue to diffusion protocol about nodes' movement is delivery of most sensor events by least overhead in reconstruction of route with low delay.

In this article, we have used learning automata which is considered as an intelligent method in order to amend inefficiency of direct diffusion towards dynamism. In next Session, We declare some related works such as One Phase Pull and Two Phase Pull etc. Learning automata structure introduced in Session three. Here, we explain Standard LA and use it in approach. Our approach is situated in session four. Session five is performance evaluation for DDLA with some parameters and finally in last session conclusion and future work proposed.

\section{RELATED WORKS}

Recently some researchers have been done on routing in wireless sensor networks. One of the famous protocols in this field is directed diffusion.

The most basic method is directed diffusion method which has been presented by Fabio Silva. This method uses three phases in its routing. Interest phase, discovery phase and choosing the best route phase. Two-Phase attraction method is a very suitable method for some of applications but in opposite it acts very weakly for some other applications. Especially, those applications in which there are many sources and receivers and receivers are such connected to each other that traffic data volume will much increased.

For solving this problem, push diffusion method [2] is brought up in which role of information diffusers and data users are changed and it causes data sources to actively look for users. Advantage of push diffusion is distraction of control data in it in order to find receives. The problem of this method is that it doesn't act well if the numbers of sources are many and numbers of receivers OR central nodes are few.

One Phase Pull method [3] was presented for improving this problem. This idea is based on requester who omits one of Phase of two Phase Pull methods. The application of this method when number of sources is many and number of receivers is few is done.

Another improvement of directed diffusion is the method based on diameter of hexagon [4]. In this method, sensor nodes are ordered as a beehive with a fixed topology and main information is sent and received on the diameter of hexagon. In fact, available hexagons are considered as basic routs of data. In order to distribute energy consumption among sensors in this method, basic routs are periodically changed. For more decrease in energy consumption, we activate sensors when sending data and deactivate them when they are idol. It has excellent efficiency in comparison with basic diffusion when responding to an interest with regards to energy consumption rate and delay.

Another improvement is directed diffusion energy productivity by use of Passive clustering [5]. With regards to huge cost that data flooding diffusion imposes on energy resources, a method was suggested for preventing energy waste which is named passive clustering. Basic job of clusters is optimizing of flooding message exchanges which prevents it's too much overhead. Normal clustering is done by use of flooding diffusion of controlling messages when there is not any traffic in the network, therefore, it results are in energy waste for keeping cluster structure. In fact, passive clustering is a suitable mechanism for increasing efficiency of the whole network. Simulation results show that directed diffusion along with passive clustering has better performance than basic diffusion with regards to transfer rate, network density increase and delay.

Another improvement which has been realized in directed diffusion is E-Span method and LPT [6] for data density in wireless sensor networks. When an event is observed in a special zone in sensor networks, data is sent toward central node or destination by available sources in that zone. Data of these sources are usually accumulated on the route. Data density decreases communication of overhead cost and increases energy productivity.

In suggested structure, available sources in event zone form a fixed tree structure named LPT in order to ease data density. This method is used for increasing life span of sources which frequently transfer data report. In E-Span, sources which have the most remained energy are chosen as root and nodes of another source choose their parent node from their neighbors based on remained energy information and distance from root, then in LPT those nodes which have more remained energy are chosen as accumulator parent.

This method includes self-amendment method by which the tree is reconstructed whenever a node stops or a failed connection is discovered. Simulation results show that whenever LPT algorithm is used for data density in directed diffusion, source's life span is considerably increased. Another improvement is light directed diffusion [7].

For Decreasing network overhead, number of exchanged packs for periodical diffusion of interests and delivery of discovered data to central node whose result is energy consumption reduce because of decreasing exchanged controlling message and as a result increase of network life span.

Light directed diffusion [8] is based on this idea that it produces a spars logical topology by simple local regulations and then directed diffusion is executed on this topology.

\section{LEARNING AUTOMATA}

Learning Automata are adaptive decision-making devices operating on unknown random environments. A Learning Automaton has a finite set of actions and each action has a certain probability (unknown to the automaton) of getting rewarded by the environment of the automaton. The aim is to learn to choose the optimal action (i.e. the action with the highest probability of being rewarded) through repeated interaction on the system. If the learning algorithm is chosen properly, then the iterative process of interacting on the environment can be made to result in selection of the optimal action.

Fig. 1 illustrates how a stochastic automaton works in feedback connection with a random environment. Learning Automata can be classified into two main families: fixed 
structure learning automata and Variable Structure Learning Automata (VSLA). In the following, the variable structure learning automata which will be used in this Situation is described [9].

A VSLA is a quintuple $(\alpha, \beta, p, T(\alpha, \beta, p))$, where a, $\beta$, $\mathrm{p}$ are an action set with s actions, an environment response set and the probability set $p$ containing $s$ probabilities, each being the probability of performing every action in the current Internal automaton state, respectively. If the response of the environment takes binary values learning automata model is P-model and if it takes finite output set with more than two elements that take values in the interval $[0,1]$, such a model is referred to as Q-model, and when the output of the environment is a continuous variable in the interval $[0,1]$, it is refer to as S-model. The function of $\mathrm{T}$ is the reinforcement algorithm, which modifies the action probability vector $\mathrm{p}$ with respect to the performed action and received response. Assume $\beta €[0,1]$. A general linear schema for updating action probabilities can be represented as follows. Let action i be performed then (1), (2) shows as follows [10]:

$$
\begin{aligned}
& p_{i}(n+1)=p_{i}(n)+a\left[1-p_{i}(n)\right] \\
& p_{j}(n+1)=(1-a) p_{j}(n) \quad \forall j j \neq i \quad \text { Desirable (1) } \\
& p_{i}(n+1)=(1-b) p_{i}(n) \\
& p_{j}(n+1)=(b / r-1)+(1-b) p_{j}(n) \quad \forall j j \neq i
\end{aligned}
$$

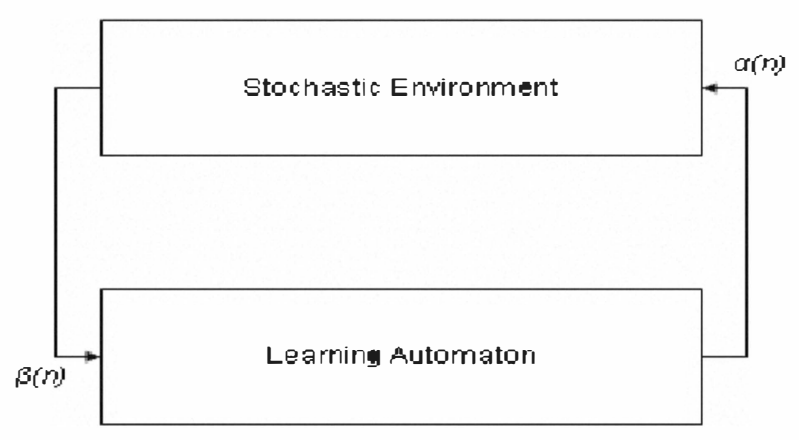

Figure 1. Learning Automata and Environment

Where $a$ and $b$ are reward and penalty parameters. When $a=b$, the automaton is called $L_{R P}$. If $b=0$ the automaton is called LRI and if $0<\mathrm{b}<<\mathrm{a}<1$, the automaton is called $\mathrm{L}_{\mathrm{R}} \boldsymbol{E}_{\mathrm{P}}$ [11].

\section{DiRECTED DifUSION LEARNING AUTOMATA(DDLA)}

Central node's movement is managed by an intermediate node in order to improve mentioned limitation in the previous section. For choosing intermediate node, central node diffuses a message for updating. It uses this message for announcing its current situation and therefore choosing a temporary intermediate node close to itself. In fact, updating message of central node is periodically diffused at a special rate.

We can also diffuse updating message not periodically and whenever central node wanted. All nodes which receive this message send query announcing becoming intermediate to central node. Central node chooses one of these nodes as intermediate and it is announced to other nodes. But, if one of these nodes was already intermediate, central node doesn't change its intermediate because it has not become far from its previous intermediate.

We want to use learning automata in directed diffusion method, from now; we have named this method DDLA. As we know, we can control route of last movement on data diffusion in sensor networks by use of learning automata. As mentioned before, in this algorithm, when considered destination (sink) moves we should be able to find stronger and more energetic route based on the request that sink sends to its neighbors and announces to them that it is moving to a new place from its current place. Here, it is enough to model last movement on learning automata and to find route by operation and environment responses.

In DD method, when receiver moves, all three phases are inevitably implemented in order to receive information from sender and this operation causes to increase overhead. Therefore, we have considered this algorithm for displacement of sink or receiver. In this algorithm, we have a phase to directed diffusion in order to compensate this deficiency. We name the created phase as covering phase which is added after routing phase.

In suggested algorithm, we have considered a machine which works based on stochastic learning automata and this machine is applied in the Phase before transfer and delivery of pack to receiver. In this method, we have to find new place of receiver when transferring information pack. For doing this, each receiver which wants to change its place from $\mathrm{i}$ to $\mathrm{j}$ should inform all it's around neighbors by sending an acknowledge query. In Basic DD, the route should be reconstructed from source (origin) to destination which causes to increase overhead and to waste energy; this has been showed in Fig. 2.

But, in suggested method, this overhead has been decreased and route is built by faster speed. Suggested algorithm solves the problem by intermediate nodes. Intermediate node is the one which considers the shortest route with the least energy from disjoint point to receiver. Here, we recognize this route by using learning automata. 


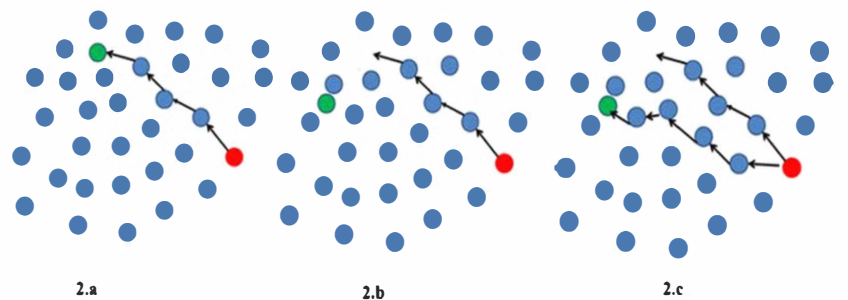

Figure 2. 2-a and 2-b showed that sink is moving, in 2.c basic directed diffusion try to run again (all three phase) to find new path from source to sink

Now, we consider ending diffusion route with the help of learning automata with actions and regional response. Region is dynamic and nod-deterministic. Number of action of each automata is equal to number of neighbors of each node. In fact, one action is defined for each node. When sink node moves, it announces its movement to its neighbors. In the first phase of this algorithm, we have to find on optimal route from transfer place of sink node to its current place. Each action is equal to choosing a neighbor based on probability of its choosing. As we know, at the beginning the probability of each action is the same. Each node which has been chosen, repeats choosing till we eventually reach to sink. Region always returns the time that takes for the pack to go and come back from current node to sink as a response. As a result, an action which receives response from sink in shorter time is more probable to be chosen. This is hierarchically repeated till eventually a route which is the fastest is chosen.

Fig. 3 shows this. Now a result is made from disjoint point to sink. In continuation, we want to create a route from sink to origin in which we have considered two factors: 1The least time duration 2- Energy. By "The least time Duration" we mean the time that pack arrives to receiver from sender in the least possible time. By energy we mean a route to be chosen that is acceptable with regards to energy and less energy is used (in order to save energy). In this Phase, node chooses an action, therefore, one of neighbors is chosen. This is hierarchically done like previous part and finally a route is made from receiver to send. Action in response will return time of sending, pack and also their energy to the region. In order to make balance between time and energy we have used below formula.

. When the result of this formula is more it means probability of that action is more. We have considered 10 coefficients of time and 5 coefficients for energy.

Time coefficient is considered negative, it means we have $\mathrm{q}=5^{*} \mathrm{E}-10$ when $\mathrm{E}$ is energy and $\mathrm{T}$ is time. Now, the action will be chosen which has higher Q. In Fig. 4 this is shown. In continuation a route between origin and destination is chosen from which packs are exchanged between origin and destination.

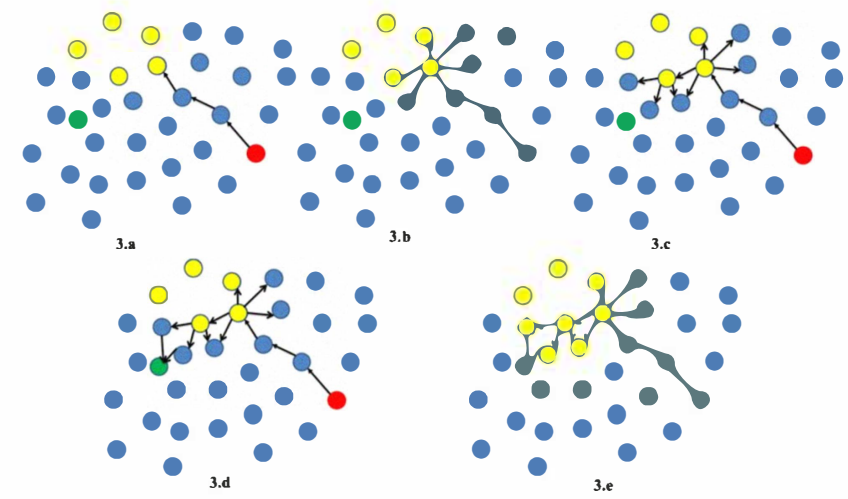

Figure 3. Implementing $p 1, \alpha 1, \beta 1$ in Sensor Network

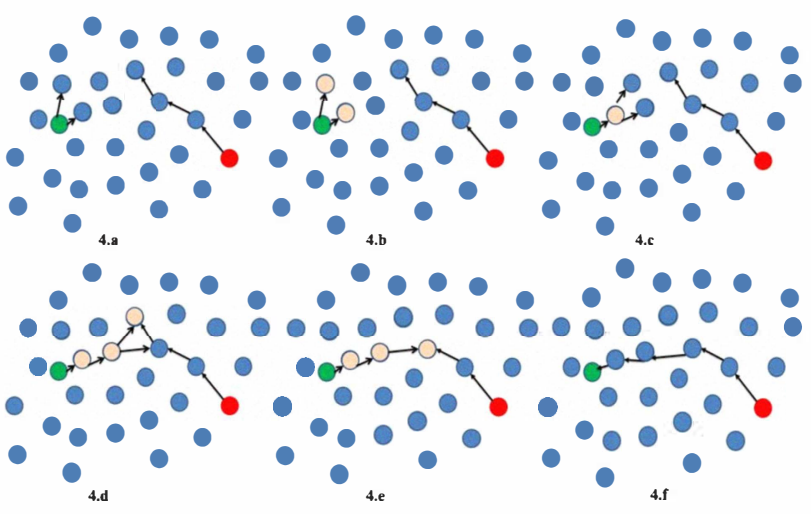

Figure 4. Implementing $\mathrm{p} 2, \alpha 2, \beta 2$ in Sensor Network

\section{SIMULATION AND COMPARISON CONCLUSION}

In this part, we see result of Simulation for each scenario which has been discussed in previous part and relevant figure is shown. For Simulation software of NS2[12,13] has been used whose model of energy is considered $0.660 \mathrm{~W}$ for sending and $0.395 \mathrm{~W}$ for receiving data which is according to energy in card of PCM-CIA-WLAN which is introduced in NS2. Then results of each Simulation have been assessed.

The important point is that we have implemented the algorithm by standard method of automata and then we have compared all of them to each other. In all methods, we turn on a chronometer and make decision based on it. So, in suggested algorithm if the time of delivered pack is less than previous mode desirable situation has been occurred but if the time becomes more, undesirable situation has occurred.

In these models, we have considered $0-2$ as reward and 0.07 as punishment. In this experiment, number of nodes is 100 and they are distributed in the area of $160 * 160 \mathrm{~m}$. Dynamism rate in experiments has been considered 20M/S, so that these speeds show the difference between improvements of our suggested method with basic diffusion. Therefore, we don't impose heavy load on the network, our goal is observing protocol behaviors under normal operational conditions.

Here, we have compared One Phase and two-Phase attraction algorithms with suggested algorithm. Compared 
parameters are Discovery Overhead, Network Traffic Rate, Event Delivery Rate and Energy.

\section{A. Comparing Discovery Overhead}

Fig. 5 shows overhead amount for basic diffusion model, One Phase Pull and suggested algorithm under different speeds of central node by stochastic movement model in a network.

In this part, we have tried to decrease data loss rate by repeating diffusion phase. But as results show, basic diffusion uses a big proportion of available traffic in the network for this overhead because of much controlling overhead which it has in diffusion phases. In One Phase Pull method, controlling overhead has been omitted due to deletion of phase of sending discovered data, but available overhead is still high.

In suggested algorithm produced overhead of packs is much less in comparison with two previous models because method of construction and amendment of routing tree is used. This outcome assures us that suggested method improves basic considerably in order to support dynamism.

\section{B. Network Traffic Rate}

Network traffic rate is much influenced by phase of directed diffusion algorithm. The less the number of these phases becomes, the less become created packs in the network and therefore network traffic rate decreases. In basic algorithm and One Phase Pull packs are produced since the beginning due to repetition of threefold phases and this is the reason of making the pack.

In suggested algorithm when central node moves, nodes choose their considered action and this prevents creation of threefold phases of basic algorithm, and this prevents creation of extra packs, like Fig. 6.

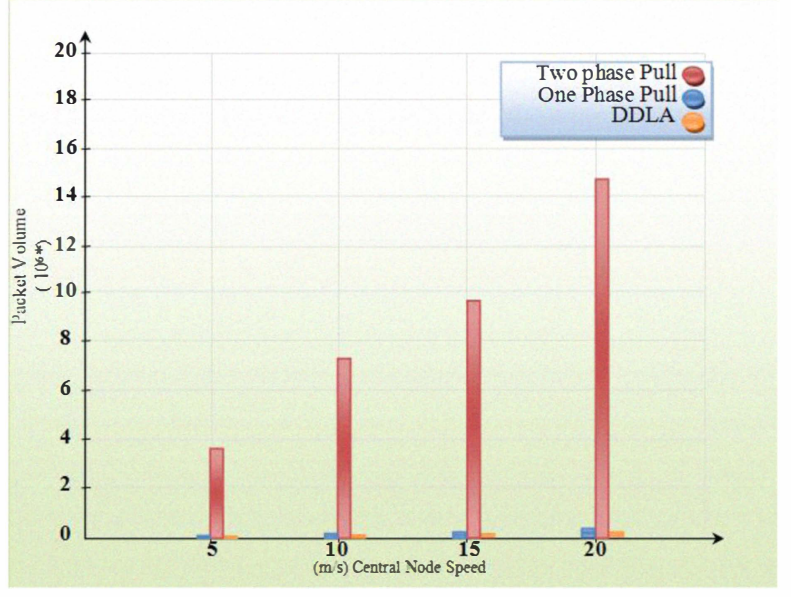

Figure 5. Comparing Between Explorer Overhead in DDLA and One and Two Phase Pull

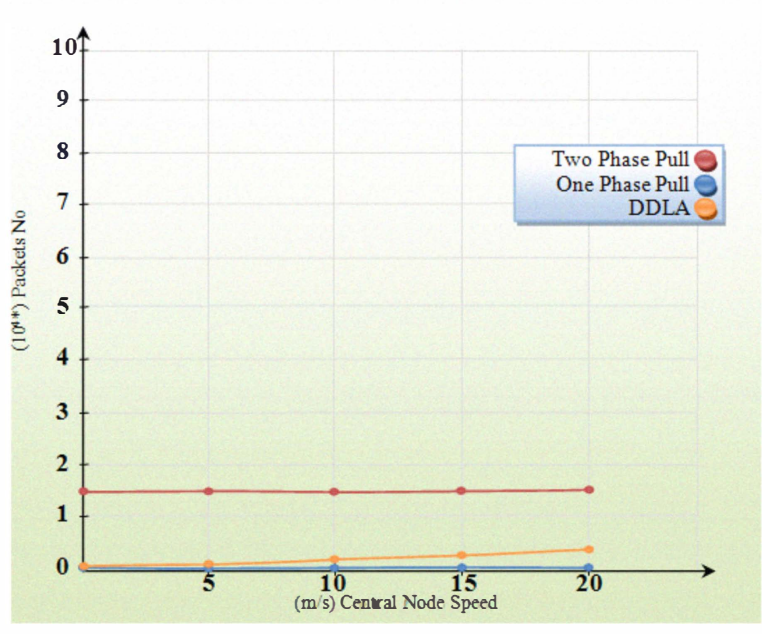

Figure 6. Movement Effect on Network Traffic Rate

\section{Event Delivery Rate}

In Fig. 7, you see Event Delivery Rate for network which includes 100 nodes. As you see, suggested algorithm keeps event delivery rate at an acceptable rate in comparison with basic diffusion. Above outcome is encouraging with regards to this fact that event delivery rate in basic diffusion decreases because of central nodes movement by different speeds. With regards to above figure, by changing speed, event delivery rate in basic diffusion decreases over $40 \%$. If $50 \%$ loss of sensed events is tolerable for an application, basic diffusion can be used for central nodes with relatively low speed.

With repetition of diffusion phases, events delivery rate of basic diffusion can be increased to some extent. But here the problem is overhead rate resulting from increasing discovery rate. Fig. 7 shows the outcome in which by movement of central node and being remote from a threshold, diffusion phases are repeated in order to reconstruct the rout and delivery of data to destination.

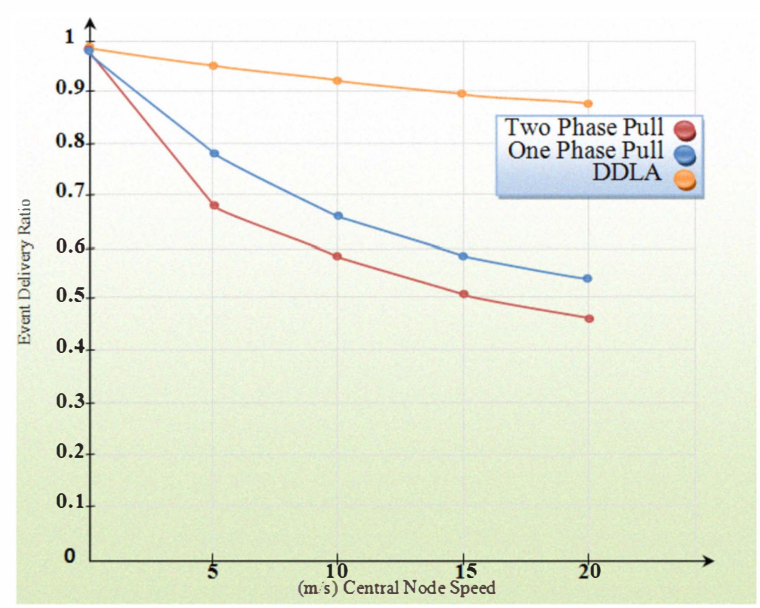

Figure 7. Movement Effect on Event Delivery Rate 
With repetition of diffusion, delivered events rate and also overhead of discovery have increased. But, Event Delivery Rate is not cost-effective with regards to produced overhead and consumed energy in basic methods.

\section{Network Consumed Energy}

All three phases of algorithm are created in two-Phase attraction algorithm and there is discovery Phase too. Therefore, in basic method sending and receiving and processing of pack are much more than One Phase Pull algorithm, thus node's energy consumption will become more. In One Phase Pull method sending and receiving operation will also become less by omitting discovery Phase and more energy will remain in network. But, in suggested algorithm saving energy is very obvious; because, three phases of two previous algorithms are not created and this has the greatest influence on saving energy. The faster the central node moves, the more difference suggested algorithm shows and its reason is producing less controlling packs than two available algorithms. Three methods are compared with regards to energy in Fig. 8.

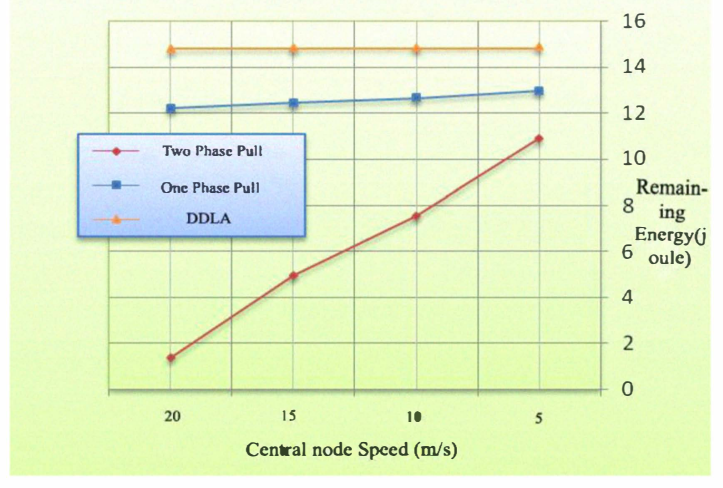

Figure 8. Moving Effect Central Node on Network Remaining Energy

\section{CONCLUSION AND FUTURE WORK}

Our assessment shows that directed diffusion is absolutely sensitive to dynamism of central nodes which move very slowly. After checking basic diffusion, we found out that this protocol doesn't do any efforts for keeping routing tree against dynamism. Therefore, when using it, no change is observed in network traffic. But in case of existence of negligible dynamism in the network, amount of event delivery will not change too much even with repetition of diffusion phases in return for node's movement and is encountered with $48 \%$ loss. Negligible change in amount of event delivery is because of delay in data delivery to the destination in base diffusion. Anyway, existence of these losses may be tolerable for some users in order to use basic diffusion. In opposite, after applying the changes to the protocol in the suggested method and with using intelligent method of learning automata amount of losses of sensed event have been considerably deceased in different speeds.
Moreover, traffic of the whole network has been reduced too much. One of future works that we can do is to make algorithm so much intelligent that it supports movement of nodes of origin too. Because, in the available current method we have considered sink's movement as a criterion and we can even present a design for the movement of intermediate in order to reconstruct last route with least waste of time and energy.

\section{REFERENCES}

[1] Ch. Intanagonwiwat, R. Govindan, D. Estrin, "Directed Diffusion: A Scalable and Robust Communication Paradigm for Sensor Networks", IEEE/ACM Transactions on Networking (TON), Vol. 11, Issue 1, ISSN: 1063-6692, pp. 2-16, 2003. DOI: http://portal .acm.org/ft gateway.cfm? $\mathrm{id}=638335 \&$ type $=$ pdf\&coll $=\mathrm{G}$ UIDE\&dl=GUIDE\&CFID=92179123\&CFTOKEN $=40425763$

[2] I.F. Akyildiz, W. Su, E. Cayirici, "Wireless sensor networks: a survey", Vol. 38, Issue 4, pp. 393-422, 2002. DOI: http://dx.doi.org/10.1016/S1389-1286(01)00302-4

[3] J. Heidemann, F. Silva, D. Estrin, "Matching Data Dissemination Algorithms to Application Requirements", Proceedings of the First ACM International Conference on Embedded Networked Sensor Systems (SenSys'03), ISBN: 1-58113-707-9, LA, USA, pp. 218-229, 2003. DOI: http://doi.acm.org/10.1145/958491.958517

[4] Y. Sh. Chen, Yau-Wen Nian, J.P. Sheu, " An Energy-Efficient Diagonal-Based Directed Diffusion for Wireless Sensor Networks", Proceedings of the 9th International Conference on Parallel and Distributed Systems(ICPADS'02),IEEE Computer Society, ISBN:07695-1760-9, USA, pp.445-460, 2002.DOI: http://doi.ieeecomputersociety.org/10.1109/ICPADS.2002.1183437

[5] A. Köpke, Ch. Frank, H. Karl, V. Handziski, W. Drytkiewicz, "Improving the Energy Efficiency of Directed Diffusion Using Passive Clustering", Proceedings of First European Workshop in Wireless Sensor Networks (EWSN), ISSN:0302-9743, ISBN: 978-3540-20825-9, Springer Berlin, pp.172-187, 2004. DOI: http://www.springerlink.com/content/d3jukem 4 phxdtm $7 \mathrm{~m} /$ fulltext.pd f

[6] M. Lee, V.W.S. Wong, "LPT for Data Aggregation in Wireless Sensor Networks", Proceedings of IEEE Global Telecommunications Conference (GLOBECOM), BC, USA, Vol. 5, pp.29-74, 2005. DOI: http://dx.doi.org/10.1109/GLOCOM.2005.1578302

[7] A. Marcucci, M. Nati, C. Petrioli, A. Vitaletti, "Directed diffusion light: low overhead data dissemination in wireless sensor networks", 61st IEEE Conference in Vehicular Technology (VTC 2005-Spring), ISSN: 1550-2252, Vol. 4, pp. 2538-2545, 2005. DOI: http://dx.doi.org/10.1109/VETECS.2005.1543793

[8] A. Marcucci, M. Nati, C. Petrioli, A. Vitaletti, "Directed diffusion light: low overhead data dissemination in wireless Sensor Networks", 61st IEEE Vehicular Technology Conference, VTC 2005-Spring, ISSN: 1550-2252, Vol. 4, pp.2538-2545, 2005. DOI: http://doi.ieeecomputersociety.org/10.1109/VETECS.2005.1543793

[9] K. S. Narendra, M. A. L. Thathachar, Learning automata: An introduction, Prentice Hall, ISBN: 0-13-485558-2, Pages: 476, 1989.

[10] H. Beigy, Intelligent Channel Assignment in Cellular Networks: A learning Automata Approach, A Dissertation for Doctor of Philosophy in CS Amirkabir University, Tehran, Iran, 2004.

[11] A.S. Poznyak, K. Najim, Learning Automata and Stochastic Optimization, ISBN 3-540-76154-3 Springer-Verlag Berlin Heidelberg New York, 1997.

[12] D. Ganesan, R. Govindan, S. Shenker, D. Estrin, "Highly Resilient Energy-efficient Multi path Routing in Wireless Sensor Networks", Proceedings of ACM MOBIHOC, ISSN:1559-1662, Vol.5, Issue 4, pp. 11-24, 2001. DOI: http://doi.acm.org/10.1145/509506.509514

[13] Ns - network simulator version 2, http://www.isi.edu/nsnam 